\title{
Immune System Findings Domain
}

National Cancer Institute

\section{Source}

National Cancer Institute. Immune System Findings Domain. NCI Thesaurus. Code C102649.

A subject domain utilized for the submission of information encompassing and representing data, vocabulary or records related to immune system findings. 